\title{
Arquitectura de acoplamiento entre INS/GPS para navegación precisa en trayectorias establecidas
}

\author{
Coupling architecture between INS / GPS for precise \\ navigation in established trajectories
}

\author{
Mónica Zabala, Lorena Cuenca, Jorge León y Fausto Cabrera
}

\begin{abstract}
A prototype based on development cards for the coupling of the inertial navigation system with GPS is implemented to improve the accuracy of navigation on a trajectory. Initially it is necessary to calibrate the inertial measurement unit (IMU) using the MADGWICK filter to obtain reliable raw data, the coupling of the global positioning system (GPS) and IMU signals is done through the Tight Coupling architecture using the Kalman filter to concurrently eliminate correlated errors between systems and achieve better accuracy in the generally navigation solution used for unmanned aerial vehicle (UAV) applications. The final application, called LJLAB, is developed in Matlab ${ }^{\circledR}$ with the aim of processing and visualizing the measured data of both systems (independent and coupled). The application is capable of showing the error in positioning accuracy that exists between technologies being statistically verified through the experimental method ANOVA which computes the absolute error between the real points and those measured through the analysis of the mean and variance of the observed data.
\end{abstract}

Index Terms-Global Positioning System, Inertial Measuremnet Unit, Inertial Navigation System, Coupling System, Sensors, Kalman Filter, Madgwick Filter.

Resumen-Se implementa un prototipo basado en tarjetas de desarrollo para el acoplamiento del sistema de navegación inercial con GPS para mejorar la precisión en la navegación sobre una trayectoria. Inicialmente es necesario la calibración de la unidad de medición inercial (IMU) mediante un filtro de MADGWICK para que los datos brutos extraídos sean fiables, el acoplamiento de las señales del sistema de posicionamiento global

M. Zabala. Investigador en el proyecto de investigación "Diseño y construcción de un Quadcopter controlado por Raspberry PI para futuras aplicaciones industriales", Escuela Superior Politécnica de Chimborazo, Facultad de Informática y Electrónica, Escuela de Ingeniería Electrónica en Telecomunicaciones y Redes, Panamericana Sur Km 1 1/2, Riobamba Ecuador, Áreas de interés navegación por satélites, posicionamiento por sensores, posicionamiento indoor, técnicas de precisión en la navegación, mzabala@espoch.edu.ec

L. Cuenca, J. León. Ingenieros en la Escuela de Ingeniería en Electrónica, Telecomunicaciones y Redes de la Facultad de Informática y Electrónica, Escuela Superior Politécnica de Chimborazo. Riobamba - Ecuador, cuencasaenz_lorena@hotmail.com,jal_c17@hotmail.com

F. Cabrera. Investigador/Responsable del proyecto de investigación "Diseño y construcción de un Quadcopter controlado por Raspberry PI para futuras aplicaciones industriales", Escuela Superior Politécnica de Chimborazo, Facultad de Informática y Electrónica, Escuela de Ingeniería Electrónica en Control y Redes Industriales, Panamericana Sur Km 1 1/2, Riobamba - Ecuador, fausto.cabrera@espoch.edu.ec
(GPS) e IMU se realiza a través de la arquitectura Tight Coupling por medio del Filtro de Kalman con el fin de eliminar errores correlacionados entre sistemas y alcanzar mejor precisión en la solución de navegación generalmente utilizada para aplicaciones en vehículos aéreos no tripulados (UAV). Se desarrolla la aplicación final denominada LJLAB en Matlab, cuya función es procesar y visualizar los datos medidos de ambos sistemas en forma independiente $y$ acopladas al mostrar gráficamente el error en la precisión de posicionamiento que existe entre tecnologías, lo cual es comprobado estadísticamente a través del método experimental ANOVA que calcula el error absoluto entre los puntos reales y los medidos a través del análisis de la media y varianza de los datos observados.

Palabras Claves-Sistema de Posicionamiento Global, Unidad de Medición Inercial, Sistema de Navegación Inercial, Arquitecturas de acoplamiento, Sensor, Filtro de Kalman, Filtro de Madgwick.

\section{INTRODUCCIÓN}

OS sistemas de posicionamiento, en la actualidad y de

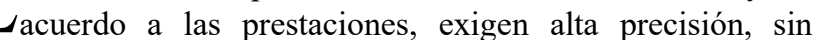
embargo, el servicio de posicionamiento estándar del sistema de posicionamiento global (Global Positioning System - GPS) es impreciso debido a factores externos que provocan la atenuación de la señal durante su propagación [1]. Existen técnicas de corrección para mejorar la precisión que incluye post-proceso [2] y correcciones en tiempo real. Los sistemas de navegación inercial (Inertial Navigation System - INS) son la alternativa para la navegación y posicionamiento en entornos interiores (i.e., indoor), a través de estos sistemas es posible eliminar errores correlacionados entre ambos sistemas, así mismo, éste puede proporcionar una solución de posición y velocidad incluso durante la interrupción de la señal de los satélites de posicionamiento por un periodo limitado. La implementación de un prototipo que ensamble ambos sistemas para la navegación de trayectorias que reduzca el error de posicionamiento se logra a través del desarrollo del algoritmo de la arquitectura de acoplamiento mediante el filtro de Kalman con lo que se obtienen resultados comparables a dispositivos de gama alta que cumplen con la misma función, pero a un precio de adquisición superior. 


\section{A. Sistema de Navegación Inercial (INS)}

La navegación inercial se basa en principios de la cinemática que al partir de un punto de posición inicial logra calcular posiciones futuras en todo momento, con el conocimiento de la velocidad, orientación y aceleración.

$\mathrm{Su}$ funcionamiento continuo brinda la solución de navegación a alta frecuencia de muestreo de al menos $50 \mathrm{~Hz}$, bajo ruido en periodos cortos de observación y extracción de datos brutos de altitud, rotación, aceleración que permite el cálculo continuo mediante la estimación de la posición y velocidad [3] basado en las mediciones iniciales.

\section{B. Sistema de Posicionamiento Global (GPS)}

GPS brinda la ubicación de un receptor en cualquier punto terrestre con disponibilidad 24 horas. La constelación está compuesta por 24 satélites distribuidos en 6 órbitas a inclinación de $55^{\circ}$ como se muestra en la Fig. 1. Generalmente los sistemas de posicionamiento global están sujetos a errores debido a la sincronización entre los relojes de los satélites y receptores, errores debido a las características de las capas de la atmósfera, errores insertados debido al multipath del área local de la antena, así como también los errores producidos por características propias del receptor como el ruido termino entre otras. Todos estos factores afectan a la solución final de posicionamiento del receptor. A través de la corrección de las medidas es posible mitigar el impacto de la imprecisión sobre la solución de navegación. Los errores previos a las correcciones se conocen como errores brutos, y los errores después del proceso de corrección se conocen como errores residuales.

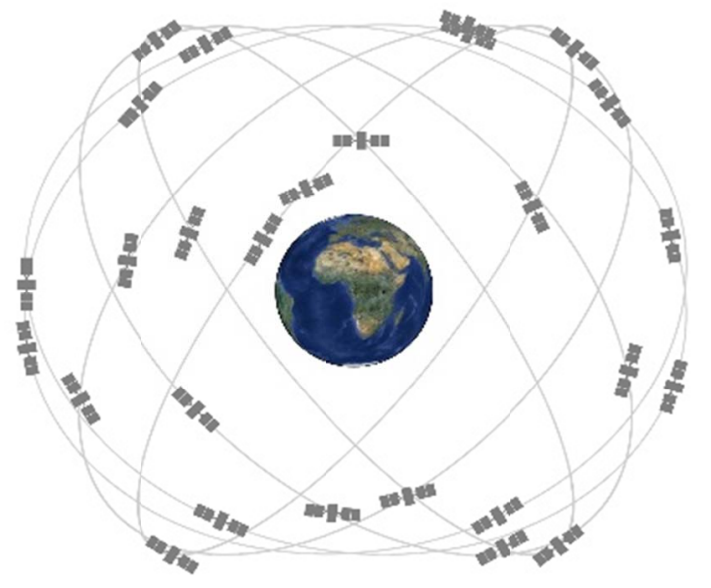

Fig. 1. Constelación de satélites GPS

\section{GPS/INS}

GPS tiene como ventaja el proporcionar una alta precisión de posición a largo plazo con errores residuales que afectan la solución final de posicionamiento a unos pocos metros con frecuencia de muestreo de $1 \mathrm{~Hz}$.

Las señales también están sujetas a obstrucción e interferencia, por lo que no se puede confiar en los receptores GPS para obtener una solución de navegación continua, a lo contrario, el sistema de navegación inercial tiene una frecuencia de muestreo de al menos $50 \mathrm{~Hz}$ y exhibe bajo ruido a corto plazo. También proporciona medidas efectivas de velocidad angular y la aceleración, así como la posición y la velocidad. Sin embargo, la precisión de una solución de navegación inercial se degrada con el tiempo.

Los beneficios del sistema de navegación inercial y del sistema de posicionamiento global GPS se complementan, por lo que, al integrarlos, las ventajas de ambas tecnologías se combinan para ofrecer una solución de navegación completa continua y con una alta precisión [4].

\section{Metodología}

El objetivo principal de la implementación es acoplar ambos sistemas para reducir el error que se presenta en los dispositivos GPS respecto a la precisión de posicionamiento en los parámetros de longitud y latitud, para ello es necesario incorporar sensores inerciales a través de una arquitectura de acoplamiento denominada tight coupling, la misma que se detalla en la Fig 2., consiste en obtener las medidas de ambos sistemas en forma independiente para posteriormente acoplarlas a través del Filtro de Kalman y obtener la solución de navegación, al utilizar tecnología de bajo costo [4].

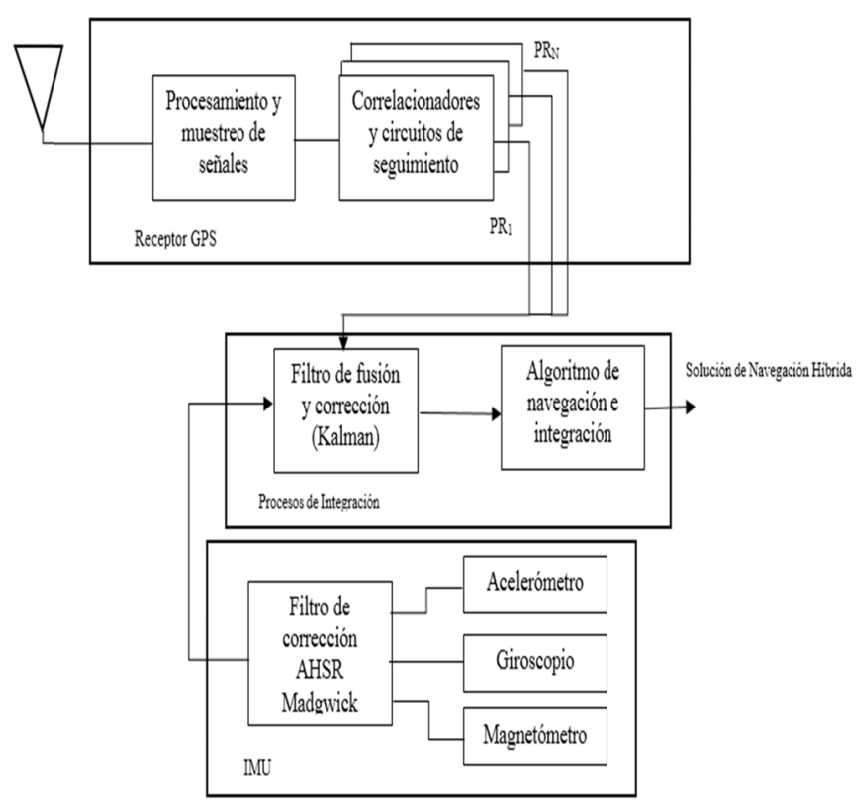

Fig. 2. Arquitectura Tight Coupling modificado

\section{A. Calibración de equipos y extracción de datos brutos}

La extracción de datos brutos del receptor GPS NEO 6MV2 [5] se realiza con tarjetas de desarrollo de hardware libre Ver Fig. 3, al utilizar las herramientas de programación propias del dispositivo para la ejecución de las líneas de comandos en la consola que permita la activación de la antena y extracción de los datos de interés en este caso la latitud, longitud y altura como se muestra en la Fig. 4. 


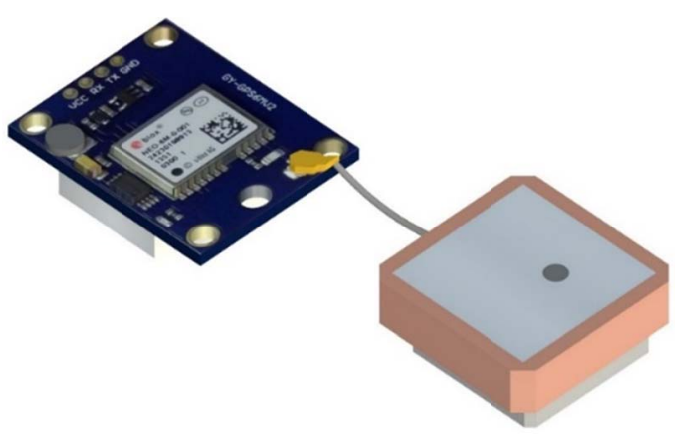

Fig. 3. GPS NEO 6MV2

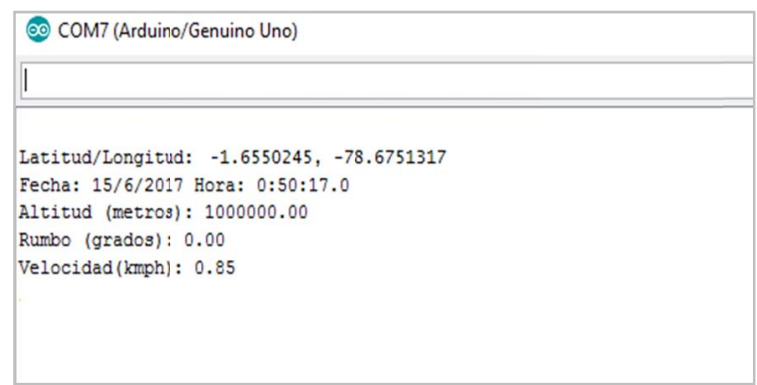

Fig. 4. Extracción de datos brutos GPS NEO6M

\section{B. Unidad de medición inercial (IMU)}

La unidad de medición inercial (IMU) utilizado es MPU 9250 (Ver Fig. 5), que integra sensores como acelerómetro, magnetómetro y giroscopio los mismos que deben ser calibrados (Ver Fig. 6), previos a su utilización para extraer los datos de manera precisa y que permita la manipulación de datos confiables.
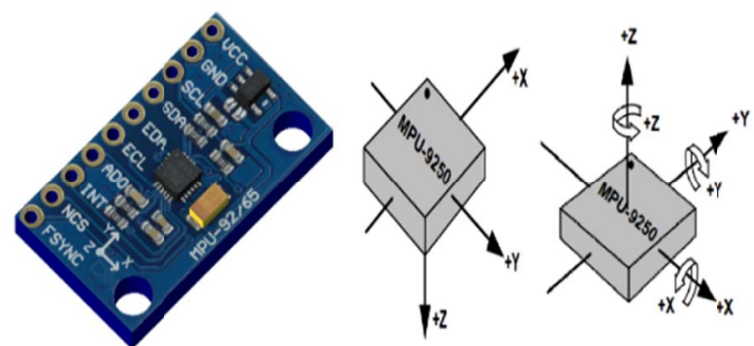

Fig. 5. Ejes MPU 9250

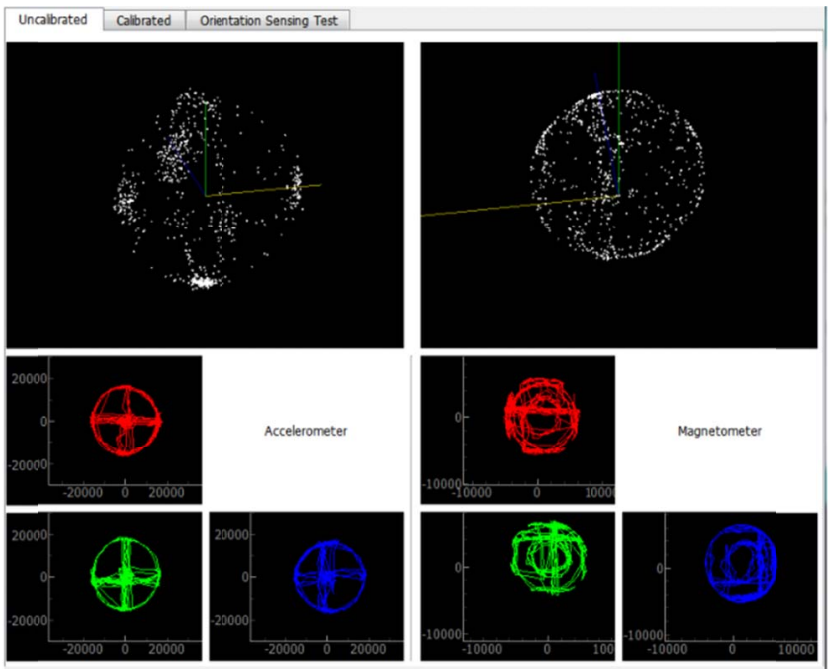

Fig. 6. Gráficas de calibración.

Para la calibración los movimientos a seguir son los mostrados en la Fig. 7, con el fin de evitar errores en las medidas obtenidas.
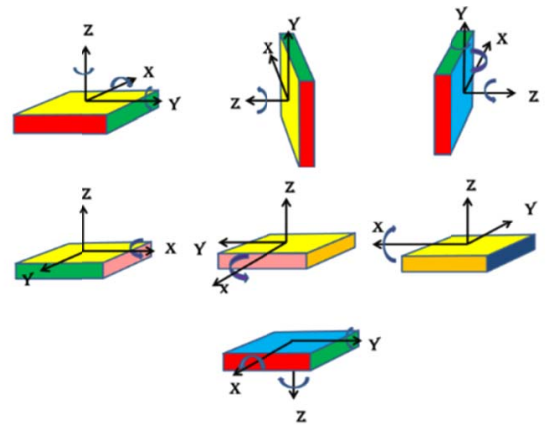

Fig. 7. Pasos de calibracion del IMU

\section{Selección de filtro de Madgwick}

Para la selección del algoritmo de fusión sensorial se opta por el filtro AHRS de Sebastián Madgwick, el cual es de código abierto, los resultados obtenidos por este filtro en este caso poseen menos deriva y sus datos son más estables como se observa en la Fig. 8, a continuación, se muestran los resultados obtenidos por cada filtro en donde se aprecia claramente que la mejor opción es el filtro AHRS [6].

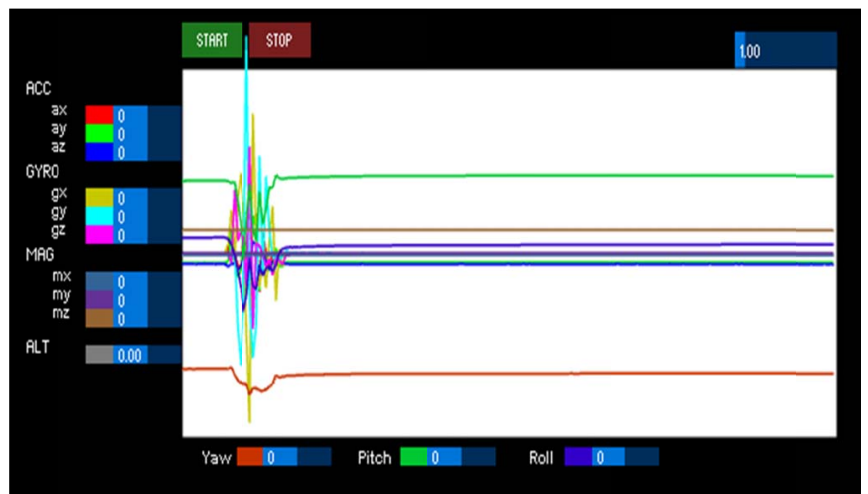

Fig. 8. Datos de sensores con filtro AHRS de Sebastián Madgwick. 
Para analizar el efecto de la aplicación del filtro AHRS de Sebastián Madgwick, se analiza la Fig. 8 en donde se puede notar que después de someter al IMU a movimientos bruscos ésta se estabiliza y elimina posibles perturbaciones.

\section{Filtro de Kalman}

El filtro de Kalman hace uso de una dimensión temporal que consiste en el análisis de la dinámica de las variables de estado y también utiliza una dimensión transversal de donde obtiene las mediciones en intervalos de tiempo de las variables observables. El filtro de Kalman es un algoritmo recursivo en el que el estado inicial es considerado una valorable aleatoria Gaussiana. Un modelo estocástico lineal es modelado gracias a las ecuaciones de transición y de medida [7]:

$$
\begin{aligned}
& \mathbf{x}_{\mathrm{k}}=\mathbf{A}_{k-1} \mathbf{x}_{\mathrm{k}-1}+\mathbf{B}_{k-1} \mathbf{u}_{\mathrm{k}-1}+\mathbf{w}_{\mathrm{k}-1} \\
& \mathbf{z}_{\mathrm{k}}=\mathbf{H}_{k} \mathbf{x}_{\mathrm{k}}+\mathbf{v}_{\mathrm{k}}
\end{aligned}
$$

donde $\mathbf{w}_{k}$ es ruido blanco $N\left(0, Q_{k}\right)$ en el instante $k, \mathbf{v}_{k}$ es ruido blanco $N\left(0, R_{k}\right)$ en el instante $k, \mathbf{A}$ es la matriz de transición de estados, $\mathbf{x}_{\boldsymbol{k}}$ es el vector de estados a estimar, $\mathbf{B}$ y $\mathbf{u}_{k-1}$ es la matriz de comandos y $\mathbf{H}_{k}$ es la matriz de observación.

Para establecer las ecuaciones del filtro de Kalman las siguientes hipótesis son requeridas.

- Las medidas de ruido son consideradas gaussianas e independientes una de otra.

- La matriz de covarianza es conocida.

- El estado inicial $x_{0}$ es un vector gaussiano independiente de $\mathbf{w}_{k}$ y $\mathbf{v}_{k}$.

El filtro de Kalman se describe en dos pasos la predicción y corrección.

1) Predicción

- Estimación a priori:

$$
\hat{\mathbf{x}}_{\mathrm{k} \mid \mathrm{k}-1}=\boldsymbol{\Phi}_{\mathrm{k}} \mathbf{x}_{\mathrm{k}-1 \mid \mathrm{k}-1}
$$

- Covarianza del error estimado

$$
\mathbf{P}_{k \mid k-1}=\boldsymbol{\Phi}_{k} \mathbf{P}_{k-1 \mid k-1} \boldsymbol{\Phi}_{k}^{T}+\mathbf{Q}_{k}
$$

2) Corrección

- Innovación:

$$
\tilde{\mathbf{y}}_{k}=\mathbf{z}_{k}-\mathbf{H}_{k} \hat{\mathbf{x}}_{k \mid k-1}
$$

- Ganancia de Kalman:

$$
\mathbf{K}_{k}=\mathbf{P}_{k \mid k-1} \mathbf{H}_{k}^{T}\left(\mathbf{H}_{k} \mathbf{P}_{k \mid k-1} \mathbf{H}_{k}^{T}+\mathbf{R}_{k}\right)^{-1}
$$

- Estación a posteriori:

$$
\hat{\mathbf{x}}_{k \mid k}=\hat{\mathbf{x}}_{k \mid k-1}+\mathbf{K}_{k} \tilde{\mathbf{y}}_{k}
$$

- Covarianza de error estimado a posteriori:

$$
\mathbf{P}_{k \mid k}=\left(\mathrm{I}-\mathbf{K}_{k} \mathbf{H}_{k}\right) \mathbf{P}_{k \mid k-1}
$$

donde $\boldsymbol{\Phi}_{\mathrm{k}}$ es la matriz de transición de estados que relación $\mathbf{x}_{k \mid k-1}$ con $\mathbf{x}_{k-1 \mid k-1}, \mathbf{P}_{k \mid k-1}$ es la covarianza del error asociada a la estimación a priori, $\mathbf{z}_{k}$ es el vector de mediciones al instante $k$, $\mathbf{H}_{k}$ es la matriz de observación que relaciona entre mediciones y el vector de estado en el instante $k$ y $\mathbf{R}_{k}$ es la matriz de covarianza del ruido.

\section{E. Filtro de Madgwick}

Es importante conocer que los ángulos de posición se pueden obtener mediante la integración de la velocidad angular que no es más que la derivada del ángulo en el tiempo.

$$
\theta(t)=\int \omega(t) d t+C
$$

donde $C$ es la constante de integración y es responsable de la presencia de errores en la medición que son de carácter acumulativo, por tal motivo se ha realizado la implementación del filtro AHRS de Sebastián Madgwic.

Los valores de actitud yaw, pitch y roll, aparte de ser representados gráficamente por medio de la aplicación en Processing, también se muestran numéricamente, sin embargo, es en la representación gráfica en donde se puede encontrar infinidad de aplicaciones, pues permiten el monitoreo de la posición de los cuerpos.

\section{RESULTADOS}

Para las mediciones se elige una trayectoria definida en la Fig. 9 dentro de la Escuela Superior Politécnica de Chimborazo (ESPOCH), la cual se ha dividido en 35 puntos los cuales serán evaluados realizar su respectivo análisis [8].

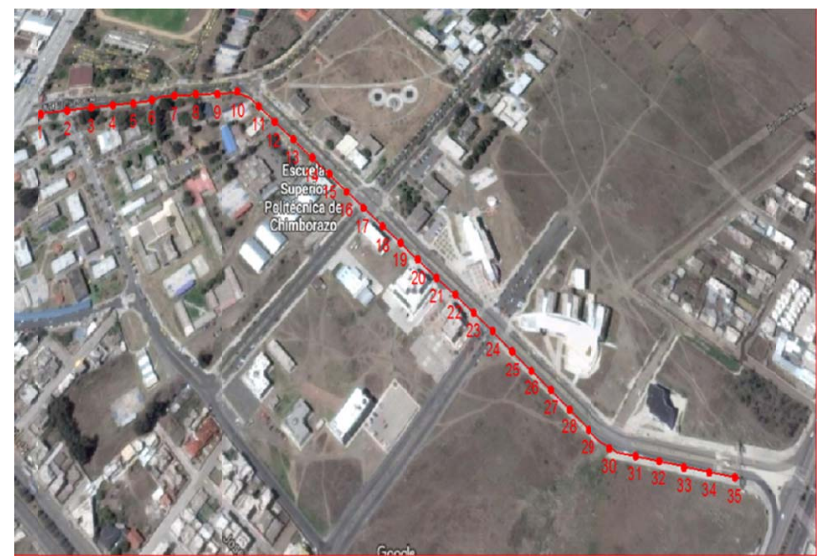

Fig. 9. Trayectoria dentro de la ESPOCH.

\section{A. Validación de resultados mediante métodos estadísticos}

El diseño experimental es una herramienta científica muy poderosa de la estadística, la cual consigue analizar los resultados de metodologías diferentes, en función de factores de variabilidad para con ello determinar la influencia de dichos factores en las variables respuesta. Se define dos métodos para su posterior análisis estadístico, el método inicial hace referencia al dispositivo GPS NEO 6M y el segundo al sistema inercial integrado con GPS. El análisis estadístico se centra en la evaluación del parámetro de precisión alcanzada por los dos sistemas de navegación en forma independiente (GPS) y fusionado (GPS+IMU). El análisis de varianza (ANOVA) [9], permite obtener el error 
existente por parte de las mediciones proporcionadas por los dos métodos, con ello se determinará la diferencia existente entre los resultados de los sistemas.

\section{B. Método experimental}

Se plantea y organiza el trabajo experimental en 4 pasos que comprende [8]:

- Identificación del problema: determinar la existencia significativa de precisión entre sistemas

- Elección de la variable de respuesta: la distancia existente entre la coordenada real y la coordenada medida.

- Influencia con la variable respuesta: el factor de bloque a evaluar es la velocidad del dispositivo en el momento de tomar la medición,

- Escenario: se experimentará con dos velocidades diferentes por cada método 40 y $208(\mathrm{~m} / \mathrm{min})$.

En [10] se muestran los datos del ejercicio experimental con los cuales se parte para su análisis con la tabla ANOVA, se muestra el error calculado en centímetros a partir de la distancia existente entre las coordenadas proporcionadas por los dos métodos y las coordenadas reales de cada uno de los 35 puntos mostrados. Para determinar las variaciones la distancia que existe entre los dos métodos a usarse se debe utilizar la fórmula de distancia entre dos puntos geográficos para determinar el error absoluto.

$$
\begin{aligned}
& d=\left(6371 * \cos ^{-1}(\cos (90-\text { Lat } 1) * \cos (90-\text { Lat } 2) \ldots\right. \\
& \ldots+\sin (90-\text { Lat } 1) * \sin (90-\text { Lat } 2) * \ldots \\
& \ldots * \cos (\text { long } 1-\text { long } 2))+100000
\end{aligned}
$$

Luego de obtener el error absoluto de cada una de las 35 observaciones, para los dos métodos y para los 3 factores de bloque, se procedió a calcular la media de cada una resumido en la Tabla I.

TABLA I

ERROR MEDIO (CM) DEL FACTOR PRINCIPAL Y FACTOR DE BLOQUE

\begin{tabular}{cccc}
\hline \hline \multirow{2}{*}{ Método } & \multicolumn{3}{c}{ Velocidad $(\mathrm{m} / \mathrm{min})$} \\
& 40 & 160 & 280 \\
\hline GPS & 92.5 & 109.5 & 340.4 \\
GPS+IMU & 5.5 & 8.3 & 10.7 \\
\hline \hline
\end{tabular}

Para realizar la comparación entre los dos sistemas, es necesario plantear la hipótesis que permita evaluar el rendimiento del sistema respecto al parámetro de precisión, así la hipótesis nula plantea que no existe diferencia significativa entre los dos sistemas, mientras que la hipótesis alternativa plantea la existencia de una diferencia significativa. Afirmación que es establecida mediante la comparación de media y varianza. Se muestra en la Tabla II la estructura que posee el ANOVA en un diseño DBCA (Diseño de Bloque Completamente al Azar) [11].

\begin{tabular}{|c|c|c|c|c|c|}
\hline $\begin{array}{l}\text { Fuente de } \\
\text { variabilidad }\end{array}$ & $\begin{array}{l}\text { Suma de } \\
\text { cuadrados }\end{array}$ & $\begin{array}{c}\text { Grados } \\
\text { de } \\
\text { libertad }\end{array}$ & $\begin{array}{l}\text { Cuadrado } \\
\text { medio }\end{array}$ & $\begin{array}{c}\text { F (Estadístico } \\
\text { de prueba) }\end{array}$ & F crítico \\
\hline Tratamientos & $\mathrm{SC}_{\text {TRAT }}$ & k-1 & $\mathrm{CM}_{\mathrm{TRAT}}$ & $F_{o}=\frac{C M_{T R A T}}{C M_{E}}$ & $\mathrm{P}(\mathrm{F}>\mathrm{Fo})$ \\
\hline Boques & $\mathrm{SC}_{\mathrm{B}}$ & b-1 & $\mathrm{CM}_{\mathrm{B}}$ & $F_{o}=\frac{C M_{T R A T}}{C M_{B}}$ & $\mathrm{P}(\mathrm{F}>\mathrm{Fo})$ \\
\hline Error & $\mathrm{SC}_{\mathrm{E}}$ & $(\mathrm{k}-1)(\mathrm{b}-1)$ & $\mathrm{CM}_{\mathrm{E}}$ & & \\
\hline Total & $\mathrm{SC}_{\mathrm{r}}$ & N-1 & & & \\
\hline
\end{tabular}

TABLA II

ESTRUCTURA ANOVA PARA DBCA

Las variables necesarias para el cálculo dependen de la media global, y los totales tanto por tratamiento como por bloque, se procede a su cálculo y se muestra los valores en la Tabla III.

TABLA III

TOTAL POR TRATAMIENTO Y POR BLOQUE

\begin{tabular}{ccccc}
\multicolumn{5}{c}{ TOTAL POR TRATAMIENTO Y POR BLOQUE } \\
\hline \multirow{2}{*}{ Método } & \multicolumn{4}{c}{ Velocidad $[\mathrm{m} / \mathrm{min}]$} \\
& 40 & 160 & 280 & Total \\
\hline GPS & 92,5 & 127,5 & 146,9 & $Y_{1}=366,9$ \\
GPS+IMU & 5,5 & 8,3 & 10,7 & $Y_{2}=24,4$ \\
Total & $Y_{\bullet 1}=98,0$ & $Y_{\bullet 2}=135,8$ & $Y_{\bullet 3}=157,5$ & Total global \\
&
\end{tabular}

Los resultados deducidos en la Tabla III son utilizados para el cálculo de ANOVA.

\begin{tabular}{cccccc}
\multicolumn{5}{c}{ TABLA IV } \\
ANOVA \\
\hline \hline $\begin{array}{c}\text { Fuente de } \\
\text { variabilidad }\end{array}$ & $\begin{array}{c}\text { Suma de } \\
\text { cuadrados }\end{array}$ & $\begin{array}{c}\text { Grados } \\
\text { de } \\
\text { libertad }\end{array}$ & $\begin{array}{c}\text { Cuadrado } \\
\text { medio }\end{array}$ & $\begin{array}{c}\text { F (Estadístico } \\
\text { de prueba) }\end{array}$ & $\begin{array}{c}\text { F } \\
\text { críti } \\
\text { co }\end{array}$ \\
\hline Métodos & 19539,6 & 1 & 19539,6267 & 62,5855523 & $\begin{array}{c}18,5 \\
1\end{array}$ \\
Velocidades & 909,4 & 2 & 454,686667 & 1,45636437 & 19 \\
Error & 624,4 & 2 & 312,206667 & & \\
Total & 21073,4 & 5 & & & \\
\hline \hline
\end{tabular}

Finalmente, los resultados mostrados en la Tabla IV, permite rechazar la hipótesis nula y aceptar la hipótesis alternativa que afirma que existe diferencia significativa entre el GPS y GPS+IMU, dado que el estadístico de prueba F, calculado al dividir el cuadrado medio tanto del método como del bloque para el cuadrado medio del error, es mayor que el $\mathrm{F}$ crítico obtenido de la tabla $\mathrm{F}$ de Fisher [12]. En cuanto al factor de bloque evaluado, que es la velocidad, la tabla IV sugiere que este factor no influye significativamente en la variable respuesta. Esto quiere decir que el método utilizado para obtener el posicionamiento influye fuertemente en la variable respuesta, que, en el caso de este experimento, es la distancia entre el punto real y el punto medido por el dispositivo, mismo que se ha especificado como error absoluto. La comparación entre los valores de la media de GPS es de $92.5(\mathrm{~cm})$ mientras que INS/GPS en $40(\mathrm{~m} / \mathrm{min})$ registra la media de $5.5(\mathrm{~cm})$, la diferencia en la media del error de posicionamiento es de $87(\mathrm{~cm})$ lo cual es significativamente crítico para aplicaciones en la navegación, se comprueba así la reducción de la imprecisión en el posicionamiento al acoplar el sistema de navegación por 
satélite (GPS) con sensores inerciales. Las Fig. 10 a Fig. 13 muestran los resultados de comparación de datos de dispersión de latitud y longitud de las pruebas ejecutadas, se analiza los 10 primeros puntos del total de 35 , en trayectorias de referencia, de GPS y GPS + IMU a velocidades de 40 [m/min] y 208 [m/min]. El algoritmo desarrollado permite la integración y visualización gráfica de los datos brutos de cada sistema, así como los acoplados entre los sistemas GPS e inerciales en tiempo real, con opciones de visualización de los datos de posicionamiento de la trayectoria evaluada.

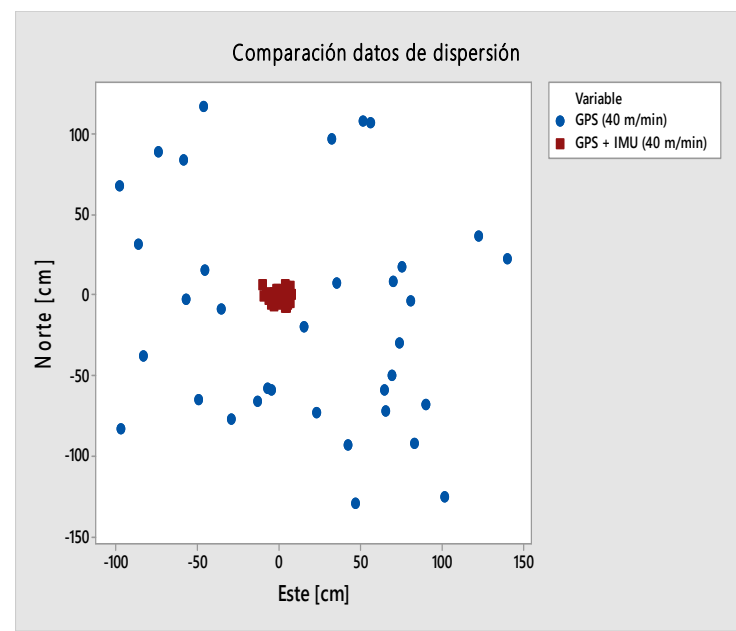

Fig. 10. GPS vs (GPS + IMU) a $40[\mathrm{~m} / \mathrm{min}]$

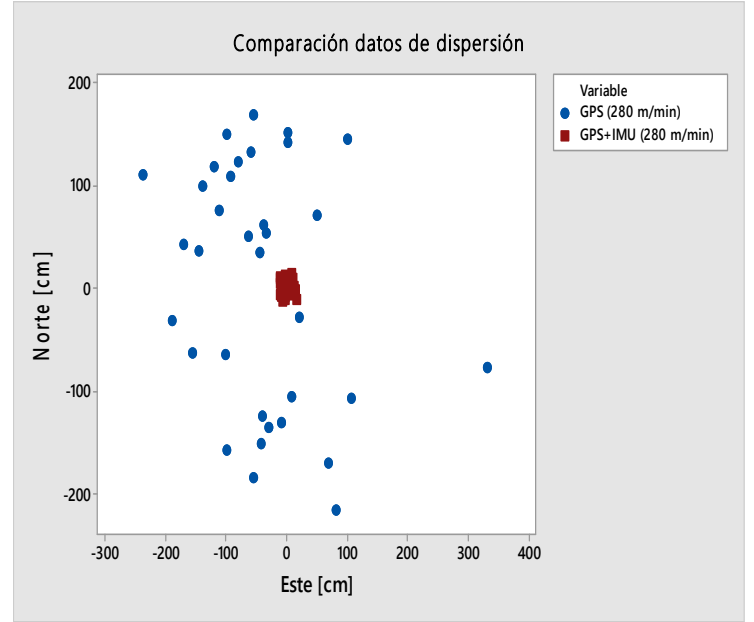

Fig. 11. GPS vs (GPS + IMU) 208 [m/min]

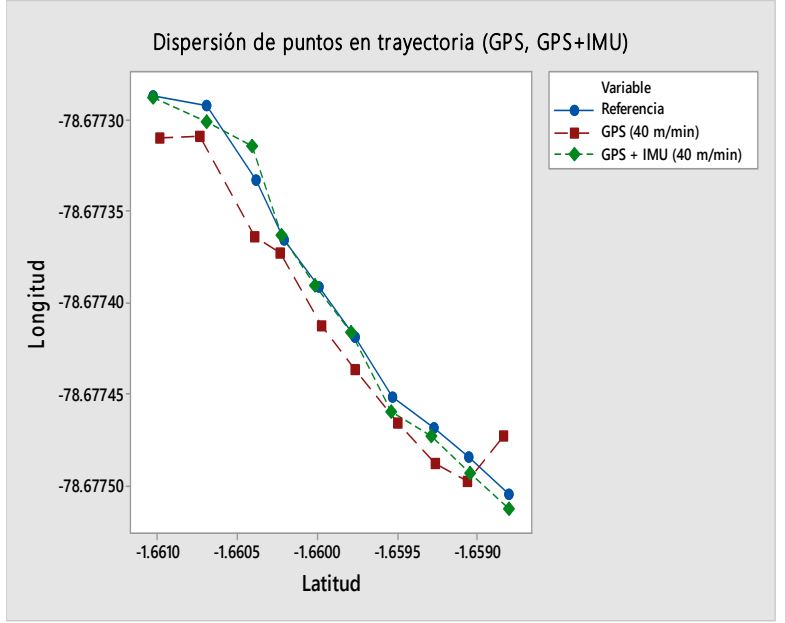

Fig. 12. GPS vs (GPS + IMU) a 40 [m/min]

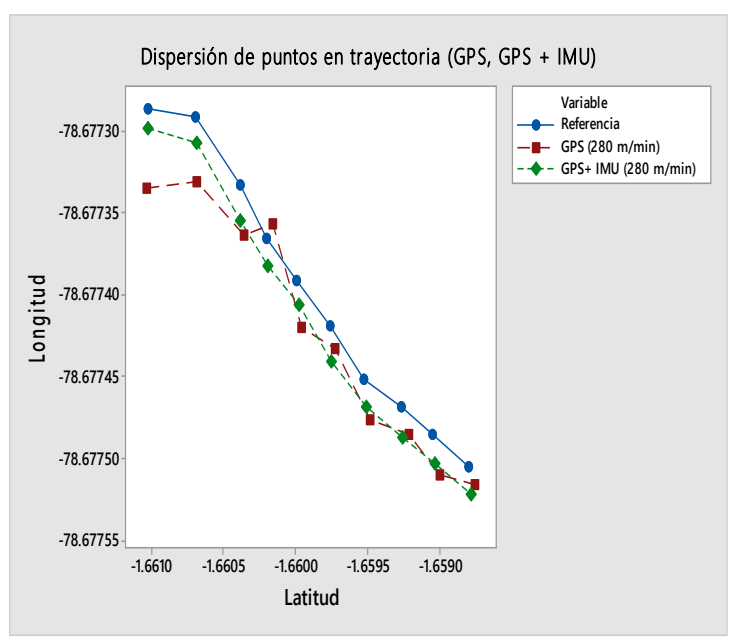

Fig. 13. GPS vs (GPS + IMU) a $280[\mathrm{~m} / \mathrm{min}]$

\section{CONCLUSIONES}

Se logró analizar el sistema de posicionamiento global al determinar los principales errores que se presentan en los equipos receptores GPS, además se selecciona la arquitectura de acoplamiento ultra estrecho con el propósito de implementar a la tecnología GPS al sistema de navegación inercial a través del filtro de Kalman. Los componentes seleccionados para la implementación del prototipo constan del módulo GPS NEO 6M, el sensor IMU MPU 9250 y la placa Arduino MEGA ya que por sus características tecnológicas y económicas se ajustan al objetivo de implementar un prototipo de bajo costo con altas prestaciones respecto a la precisión de posicionamiento. La arquitectura de acoplamiento ultra estrecho permite fusionar los datos brutos y elimina errores correlacionados mediante el incremento de la precisión en la solución de navegación. El análisis de la precisión alcanzada se evalúa a través de la media y varianza de las coordenadas medidas a lo largo de la trayectoria establecida, al calcular el error absoluto entre las coordenadas de reales y las medidas. 


\section{REFERENCIAS}

[1] Consentino, R.J. Diggle D.W. "Understanding GPS Principles and Applications", $2^{\text {nd }}$ Edition, Boston London, Artech House Publishers, 2006.

[2] López F., Ortega A., "Análisis e Implementación del diferencial de GPS con configuración simple y doble, para mejorar la precisión de un receptor GPS en la ciudad de Riobamba", Tesis de ingeniería, Escuela de Ingeniería en Electrónica, Telecomunicaciones y Redes, Escuela Superior Politécnica de Chimborazo, Riobamba, Ecuador, Tech. Report. 2017

[3] D. Titterton, J. Wseton, "Strapdown Inertial Navigation Technology", 2nd edition IEE Radar, Sonar and Navigation series 17, London, 2004, ch 2, pp. 17-26.

[4] D. Kubrak, "Etude de l'hybridation d'un récepteur GPS avec des capteurs bas couts pour la navigation personnelle en milieu urbain", Ph.D. Thesis report, ENST, 2007.

[5] Modulo GPS GY-GPS6MV2 / NEO-6M-V2 R3, Mayo 2017. [Online.]. Disponible en: https://www.ardobot.com/gps-neo-6m-v2.html

[6] Open source IMU and AHRS algorithms. Abril 2017, [Online]. Disponible: http://x-io.co.uk/open-source-imu-and-ahrs-algorithms/

[7] "Kalman Filter", Notas de clase SP413E, Signal and Proccesing Department, École Nationale de l'Aviation Civile, Invierno, 2013.

[8] Cuenca L., León J., "Implementación de un sistema de navegación inercial, para mejorar la precisión de posicionamiento de un prototipo gps en una trayectoria dentro de la ESPOCH", Tesis de Ingeniería, Escuela de Ingeniería en Electrónica, Telecomunicaciones y Redes, Escuela Superior Politécnica de Chimborazo, Riobamba, Ecuador, Tech. Report., 2017. p.85

[9] Peter Goos, David Meintrup., "Statistics with JMP: Hypothesis Tests, ANOVA and Regression", John Willey, 2016.

[10] Cuenca L., León J., "Implementación de un sistema de navegación inercial, para mejorar la precisión de posicionamiento de un prototipo gps en una trayectoria dentro de la ESPOCH", Tesis de Ingeniería, Escuela de Ingeniería en Electrónica, Telecomunicaciones y Redes, Escuela Superior Politécnica de Chimborazo, Riobamba, Ecuador, Tech. Report., 2017. p. 89

[11] Cuenca L., León J., "Implementación de un sistema de navegación inercial, para mejorar la precisión de posicionamiento de un prototipo gps en una trayectoria dentro de la ESPOCH", Tesis de Ingeniería Escuela de Ingeniería en Electrónica, Telecomunicaciones y Redes, Escuela Superior Politécnica de Chimborazo, Riobamba, Ecuador, Tech. Report., 2017. p. 91

[12] Steven M. Kay, "Fundamentals of Statistical Signal Processing Estimation Theory, Signal Processing Series", Volume I, 1st Edition, PTR Prentice Hall, 1993. 